\title{
LA ABEJA (1862-1870) Y LA RECEPCIÓN DE LA LITERATURA ALEMANA EN ESPAÑA (I). PRESENTACIÓN E ÍNDICES PARCIALES DE LA REVISTA
}

\author{
María José Cubría de Miguel - Daniel F. Hübner \\ Universidad de Zaragoza
}

\section{1. Presentación.}

Con el auge de la prensa periódica en el siglo XIX se abre en España una nueva y capital vía de difusión para la literatura alemana. Las distintas condiciones de recepción de la prensa periódica suponen, por un lado, una ampliación del número de lectores a través de instituciones como, por ejemplo, los Ateneos, donde se recibían los periódicos y las revistas culturales, al tiempo que se hacen accesibles determinados textos que, sobre todo por su extensión, no siempre lograban ser editados en libro. Entre las diversas revistas que en las décadas centrales del siglo XIX contribuyeron a la difusión de la literatura alemana en sus páginas, como el Semanario Pintoresco Español (1836-1857) o El Museo Universal (18571869), hay que destacar de modo muy especial esta que aquí nos ocupa, La Abeja. Este trabajo pretende dar a conocer el papel que desempeñó dicha revista en la difusión de la literatura en lengua alemana en España en este periodo. Para ello se ofrecen en esta primera parte del trabajo unos índices parciales de la revista, acompañados de una breve presentación de la misma. En la segunda parte, que se publicará en el siguiente número de la Revista Hispalense de Germanística, se procederá a un estudio de los textos y autores alemanes incluidos en La Abeja en relación con su recepción en España a lo largo del siglo.

La Abeja se editó entre 1862 y 1866, para, tras un breve lapso, reanudar su publicación en 1870, conservándose hoy en día seis tomos cuya extensión oscila entre las 484 y las 378 páginas $^{1}$. La revista fue fundada por el humanista catalán Antonio Bergnes de las Casas (1801-1879), divulgador entusiasta como editor de escritores extranjeros tanto clásicos como modernos, así como de obras científicas e históricas, y que llegaría a ser Rector de la Universidad de Barcelona en 1868 y Senador del Reino en $1872^{2}$. La edición de La Abeja

\footnotetext{
${ }^{1}$ Los seis tomos tienen unas dimensiones de $315 \times 220 \mathrm{~cm}$ y constan de 484, 476, 471, 480, 476 y 388 páginas respectivamente. Por lo que se deduce de la distribución interna de los tomos, se editaban anualmente once números (a excepción del tomo VI de 1870, año en que se interrumpe la publicación). La revista se publicaba en Barcelona por la "Librería de Juan Oliveres, Editor. Impresor de S. M. Calle de escudilleres, núm 57", tal como reza en los tomos. No figuran datos sobre su precio o sobre las posibles suscripciones. La revista puede consultarse de forma completa en la Biblioteca Nacional de Madrid (Signatura D 8404).

${ }^{2}$ Sobre su persona y labor intelectual pueden verse dos monografías: Santiago Olives Canals, Bergnes de las Casas, helenista y editor, 1801-1879, Barcelona, Escuela de Filología, 1947, y más recientemente Josep Antoni
} 
era, no obstante, resultado del trabajo colectivo de una "Sociedad Literaria" que integraban, además del propio Bergnes de las Casas, el doctor en Medicina D. Miguel Guitart y Buch, el catedrático de Mineralogía y Zoología D. Antonio Sánchez Comendador, el catedrático de Física D. Antonio Rave y Juan Font y Guitart, traductor del alemán de numerosos textos literarios. De la vocación germánica de La Abeja deja clara constancia su subtítulo, Revista científica y literaria ilustrada principalmente extractada de los buenos escritores alemanes, por una sociedad literaria". Por otro lado, la lista de integrantes de la "Sociedad literaria" refleja con precisión el espíritu de la revista, en la que ocuparía un primer plano la divulgación científica en los diferentes campos del conocimiento. De ahí que el modelo citado en la introducción al primer tomo fuese precisamente el de Alexander von Humboldt y que se especificasen de este modo los objetivos de la revista:

(...) el objeto que nos hemos propuesto con esta publicación, la cual constará sobre todo de pasages [sic], más o menos ampliados, de los escritores alemanes que, llevados de su amor a la humanidad, han echado sobre sus hombros la no fácil tarea de servir de intérpretes y mediadores entre la ciencia y los que apenas la han saludado ${ }^{4}$.

Sin embargo, la revista tiene especial interés para nosotros por la labor de difusión literaria que desempeñó de forma paralela, de acuerdo con una concepción de la literatura que, tal como leemos en la citada introducción, recuerda al consabido prodesse et delectare horaciano:

Además de los artículos científicos, ocuparán parte de las columnas de esta Revista otros puramente literarios, no menos instructivos que agradables; y a este efecto elegiremos las obras más desinteresadas del pensamiento y de la poesía, las dotadas de aquella gracia moral, de aquella tranquilidad genuina de un pueblo que ha dado tantas prendas a la civilización intelectual. $^{5}$

La proporción que le corresponde a la creación literaria en La Abeja es de aproximadamente un cuarto del total de páginas de la revista, aunque variará a lo largo de los tomos. Debe advertirse, además, que buena parte de los autores y textos seleccionados no eran alemanes, quizá por la creciente dificultad de encontrar materiales adecuados en lengua alemana para la revista. Predominan, como es lógico, los textos y géneros breves, y

Clua i Serena, El humanismo en Cataluña en el siglo XIX: A. Bergnes de las Casas (1801-1879), Madrid, Clásicas, 1995.

${ }^{3}$ El nombre de La Abeja tiene su precedente en algunas revistas de contenido fundamentalmente satírico de la primera mitad del siglo, como La Abeja manresana de 1812-1813 o la Abeja española, editada en Cádiz entre 1812 y 1813 por Bartolomé José Gallardo, quien posteriormente publicará en 1814 La Abeja madrileña. Más próximo y de mayor importancia es, no obstante, el diario La Abeja, publicado entre 1834 y 1836 en Madrid como continuación de El Universal. También existía en el siglo anterior un semanario literario inglés con ese nombre, The Bee, publicado por O. Goldsmith a partir de 1759, según indica Santiago Olives Canals (1947: 253).

${ }^{4}$ Antonio Bergnes de las Casas, "Introducción", La Abeja, t. I, 1862, pág. 2. También los epígrafes de los índices que ofrecen los propios tomos de la revista dan una idea muy ajustada de los contenidos de la misma: Artes, Astronomía, Biografía, Botánica, Ciencias, Economía rural, Estadística, Filosofía, Física, Fisiología, Geografía y viajes, Geología, Higiene, Historia, Historia natural, Literatura y moral, Organología, Paleontología, Poesía, Química, Variedades, Zoología y Excerpta.

${ }^{5}$ Ibid. 
hay que destacar también que el valor instructivo de muchos de ellos radica en su carácter alegórico y moralizante. Estos textos literarios son los que se presentan en los índices que siguen, aunque pueden encontrarse referencias parciales a los contenidos de la revista en algunos estudios previos ${ }^{6}$.

La revista suspendió su publicación en 1870, incluyéndose en el último número una explicación que, sin embargo, no permite despejar con seguridad las dudas sobre las razones de esta suspensión:

Motivos poderosos e invencibles, nos obligan a suspender indefinidamente la publicación de esta Revista. Podríamos referirlos porque no son secretos para muchos que conocen el estado intelectual y moral de nuestra patria; pero su relación sería por demás triste y amarga, y preferimos callarla. Hemos luchado con fe y constancia por mucho tiempo; hemos sacrificado gustosos el reposo y los intereses para llevar adelante nuestra idea; pero rendidos, tenemos que abandonarla y enmudecer con hondo sentimiento propio y de un reducido número de personas amantes del saber que constantemente nos han alentado y que con nosotros deploran y están convencidos de que es imposible aclimatar en España publicaciones de la índole de nuestra Revista.?

No obstante, dada la situación política en esos años, en pleno sexenio revolucionario, y el carácter moderadamente progresista de Bergnes de las Casas, parece que las razones aludidas de forma tan sutil no obedecerían a presiones políticas o problemas de censura, sino que la suspensión obedecería, seguramente, a la falta de viabilidad del proyecto. En cualquier caso, hay que reseñar que la revista tuvo una duración muy estimable, sobre todo considerando su vocación fundamentalmente germánica, y, en este sentido, constituye un hito significativo en la aclimatación no sólo de la literatura, sino en general de la ciencia y cultura alemanas en la España del XIX.

\section{2. ÍNDICES PARCIALES DE LA REVISTA.}

Los índices que aquí se presentan recogen de forma exhaustiva las entradas correspondientes a los epígrafes de la revista denominados "Literatura y moral" y "Poesía". En ellos se encuentran incluidos todos los textos de creación literaria de La Abeja, que son los que interesan para nuestro análisis de la recepción de la literatura alemana en España. Además hemos incluido en los índices algunos textos de tipo ensayístico de tema filológico o estético que aparecen dispersos en otros epígrafes, como "Variedades" o "Filosofía", cuya vinculación con los contenidos literarios de la revista hace oportuna su incorporación.

La ordenación de las entradas se hace siguiendo el orden alfabético de los autores y, dentro de cada autor, según el orden de aparición en la revista de los trabajos. Se ha respetado la ortografía original a excepción de la acentuación, que se ha corregido

\footnotetext{
${ }^{6}$ Véase Santiago Olives Canals (1947: 251-255) y, sobre todo, Hans Juretschke, "La Abeja de Bergnes de las Casas o aspectos del germanismo catalán hacia mediados del siglo XIX", Miscel.lània Aramon i Serra. Estudis de llengua i literatura catalana oferts a R. Aramon i Serra en el seu setantè aniversari, I, Barcelona, Curial, 1979, págs. 313-329. Más recientemente puede verse también Brian C. Dendle, "Las traducciones de Heine en La Abeja. 1862-1867", El Gnomo. Boletín de Estudios Becquerianos, 8, 1999, págs. 69-82.
}

7 "Advertencia de los editores", La Abeja, t. VI, 1870, pág. 386. 
siguiendo las normas actuales (como se ha hecho también en las citas). Asimismo hay que advertir que en la revista, como era habitual en la época, los nombres propios aparecen habitualmente en su traducción española, por lo que las iniciales se completan por lo común con los nombres en español. Sin embargo, en todas las entradas incompletas se ha optado asimismo por añadir los nombres propios en su lengua original. En los casos en los que la identidad de los autores no se ha establecido con seguridad, se indica con un interrogante después del nombre probable. En el caso del índice de traductores, para no duplicar las entradas, sólo figura el número de referencia de los índices previos.

\section{2. 1. Trabajos sin firmar.}

1. Alix y Berenguer o la Fuente de amor, t. V, 1866, 263-266.

2. Amor constancia, En el. Comedia en un acto, t. VI, 1870, 226-231.

3. Amor patrio, El. (imitación del alemán de autor desconocido), t. II, 1863, 109-110.

4. Breviario del músico, El, t. III, 1864, 352.

5. Capilla de San Jorge, La. Leyenda ateniense, t. III, 1864, 310-312.

6. Caza del caimán, La, t. VI, 1870, 190-192.

7. Connor O`Mara. Tradición irlandesa. I, t. V, 1866, 148-152.

8. Connor O’Mara. Tradición irlandesa. II y último, t. V, 1866, 177-178.

9. Desposada de Irlanda, La, t. II, 1863, 110-112.

10. Estudios histórico-literarios. Osián y su era, t. II, 1863, 219-222.

11. Estudios morales. Carlos Seymur, o el amor filial, t. V, 1866, 219-226.

12. Estudios morales. Diario de un náufrago, t. IV, 1865, 470- 472.

13. Estudios morales. Diario de un náufrago. II, t. V, 1866, 28-32.

14. Estudios morales. Diario de un náufrago. III y último, t. V, 1866, 58-61.

15. Historia de una taza de café, t. II, 1863, 390-392.

16. Historia del corazón, Una. Vivir muriendo, t. III, 1864, 187-191.

17. Historia. Del orijen [sic] de las leyes, artes y ciencias y sus progresos en los tiempos antiguos. I, t. VI, 1870, 127-182.

18. Historia. Del orijen [sic] de las leyes, artes y ciencias y sus progresos en los tiempos antiguos. II, t. VI, 1870, 210-216.

19. Historia. Del origen de las leyes, artes y ciencias y sus progresos en los tiempos antiguos. III, t. VI, 1870, 254-259.

20. Historia. Del orijen [sic] de las leyes, artes y ciencias y sus progresos en los tiempos antiguos. IV, t. VI, 1870, 293-301. 
21. Historia. Del origen de las leyes, artes y ciencias y sus progresos en los tiempos antiguos. V, t. VI, 1870, 342-349.

22. Historia. Del origen de las leyes, artes y ciencias y sus progresos en los tiempos antiguos. VI, t. VI, 1870, 377-384.

23. Hora de sueño, Una. Aventura china, t. I, 1862, 276-280.

24. Leyenda histórica. La noche de San Bartolomé en Francia o los hugonotes. 1752, t. III, 1864, 375-384.

25. Leyenda histórica. La noche de San Bartolomé en Francia o los hugonotes. 1752, t. III, 1864, 422-428.

26. Literatura. El gobernador de la Samaritana, t. VI, 1870, 90-93.

27. Literatura. Un pastor con dos conciencias, t. VI, 1870, 56-62.

28. Literatura china. Tchin-Sing y Ju-Kiouan, t. II, 1863, 186-189.

29. Literatura oriental. I, t. III, 1864, 107-109.

30. Literatura oriental. II, t. III, 1864, 146-147.

31. Literatura oriental. III y último, t. III, 1864, 184-186.

32. María. Capítulo I y II, t. III, 1864, 225-230.

33. María. Capítulo III-IV, t. III, 1864, 267-272.

34. María. (Conclusión). Capítulo V, t. III, 1864, 303-309.

35. Mejor amigo, El, t. V, 1866, 288-293.

36. Mejor amigo, El. II y el último, t. V, 1866, 336-340.

37. Monedas de oro prestadas, Las. (Traducción del alemán), t. I, 1862, 467-474.

38. Pintor, El, t. V, 1866, 373-379.

39. Pobre diablo, El. (Imitación del inglés), t. II, 1863, 228-230.

40. Poesía. A un espectro, t. IV, 1865, 116.

41. Sueños de gloria, t. II, 1863, 346-348.

42. Veneno de los obeahs, El, t. V, 1866, 451-455.

43. Verdugo, El. (Episodio de la guerra de la independencia), t. III, 1864, 348-352.

44. Visión de Mizrah, La. Traducida de una imitación del árabe, trad. Antonio Bergnes de las Casas, t. I, 1862, 184-185.

45. Vivac, El, t. V, 1866, 342-345. 


\section{2. 2. Trabajos firmados.}

46. Aikin, [John y/o Ana Laetitia], La Montaña de la Ciencia. Alegoría, t. I, 1862, 357-359.

AIKIN: v. también BARBAULD, SEÑORA.

47. Alpenburg, [Johann NePOMUK von], La Rosa de Caldres, t. I, 1862, 319.

48. [ANACREONTE], Literatura griega. Odas de Anacreonte [incluye: Cupido prisionero; El placer del sueño; Cupido y la abeja; ;Bebamos!...], trad. Aurelio Querol, t. VI, $1870,72$.

49. Andersen, [Johann Christian], La Niña de los fósforos, trad. Antonio Bergnes de las Casas, t. I, 1862, 158-159.

50. ArgensaAl, V., El esqueleto, t. I, 1862, 434-438.

51. ARndT, E[RNESTO] [ERnSt MoRITZ], La patria del alemán, t. II, 1863, 31-32.

52. ARTEAGA, E[STEBAN] DE, Estética. Investigaciones filosóficas sobre la belleza ideal considerada como objeto de todas las artes de imitación. I, t. V, 1866, 353-359.

53. ARTEAGA, E[STEBAN] DE, Estética. Investigaciones filosóficas sobre la belleza ideal considerada como objeto de todas las artes de imitación. II, t. V, 1866, 393-398.

54. ARTEAGA, E[STEBAN] DE, Estética. Investigaciones filosóficas sobre la belleza ideal considerada como objeto de todas las artes de imitación. III, t. V, 1866, 455-458.

55. ARTEAGA, E[STEBAN] DE, Estética. Investigaciones filosóficas sobre la belleza ideal considerada como objeto de todas las artes de imitación. IV, t. VI, 1870, 25-30.

56. ARTEAGA, E[STEBAN] DE, Estética. Investigaciones filosóficas sobre la belleza ideal considerada como objeto de todas las artes de imitación. V, t. VI, 1870, 40-43.

57. ARTEAGA, E[STEBAN] DE, Estética. Investigaciones filosóficas sobre la belleza ideal considerada como objeto de todas las artes de imitación. VI, t. VI, 1870, 86-90.

58. ARTEAGA, E[STEBAN] DE, Estética. Investigaciones filosóficas sobre la belleza ideal considerada como objeto de todas las artes de imitación. VII, t. VI, 1870, 129-134.

59. ARTEAGA, E[STEBAN] DE, Estética. Investigaciones filosóficas sobre la belleza ideal considerada como objeto de todas las artes de imitación. VIII y último, t. VI, 1870, 162-164.

60. AzZ-Eddin [El MocAdDESSI], La Rosa, trad. Enrique Maupoey, t. VI, 1870, 352.

61. Baggesen, A[ugusto] [August; probable confusión con Baggesen, Jens (?)], El canto de la creación, t. II, 1863, 471-472.

62. Barbauld, Señora [Aikin, Anna laetitia], El disfraz de la naturaleza, trad. Antonio Bergnes de las Casas, t. I, 1862, 158.

63. BAST, A[MADEO] DE [AMEDEE DE], El Bancolo o la limosna de un artista, t. II, $1863,348-352$. 
64. Bergnes de las CASAS, Antonio, Juan Pablo Federico Richter. Su biografía y juicio sobre sus obras, t. I, 1862, 69-77.

65. Bergnes de las CASAs, Antonio, Juan Pablo Federico Richter. Su biografía, y juicio sobre sus obras (Conclusión), t. I, 1862, 110-117.

66. BODMER, [JOHANN JACOB], Episodio del Diluvio universal [fragmento del canto V del poema La Noachida], trad. J[osé] F[ernández] Matheu, t. VI, 1870, 231-232.

Boniface Saintine, Xavier: v. Saintine, J. B.

67. Burger, G[Odofredo] A[Ugusto] [BÜrger, GotTfried August], La caza infernal, t. II, 1863, 70-71.

68. BYron, LORD, Literatura inglesa. A María Chalworth, trad. Aurelio Queral [sic], t. V, 1866, 351.

69. Cervantes, [Miguel De], Poesía. Carta de Miguel de Cervantes captivo: A M. Vázquez, mi señor, t. III, 1864, 150-152.

70. Dante Alligheri [Alighieri], La divina comedia. El infierno. Cantos de I a $\mathrm{V}$, t. III, 1864, 453-466.

71. Dante Alligheri [Alighieri], La divina comedia. El Infierno. Cantos de VI a X, t. IV, 1865, 21-32.

72. Dante Alligheri [Alighieri], La divina comedia. El infierno. Cantos XI a XV, t. IV, 1865, 104-116.

73. Dante Alligheri [Alighieri], La divina comedia. El infierno. Cantos XVI a XX, t. IV, 1865, 126-137.

74. Dante Alligheri [Alighieri], La divina comedia. El Infierno. Cantos de XXI a $X X V$, t. IV, 1865, 167-180.

75. Dante Alligheri [Alighieri], La divina comedia. El infierno. Cantos de XXVI a $X X X$, t. IV, 1865, 206- 218.

76. Dante Alligheri [Alighieri], La divina comedia. El Infierno. Cantos de XXXI a XXXIV y último, t. IV, 1865, 303-313.

77. DANTe Alligheri [Alighieri], La divina comedia. El purgatorio. Cantos I a $\mathrm{V}$, t. $\mathrm{V}, 1866,7-19$.

78. Dante Alligheri [Alighieri], La divina comedia. El purgatorio. Cantos de VI a $X, \mathrm{t} . \mathrm{V}, 1866,37-49$.

79. Dante Alligheri [Alighieri], La divina comedia. El Purgatorio. Cantos de XI a XVI, t. V, 1866, 74-89.

80. DANTE Alligheri [Alighieri], La divina comedia. El purgatorio. Cantos de XVII a XXII, t. V, 1866, 115-130. 
81. Dante Alligheri [Alighieri], La divina comedia. El purgatorio. Cantos de XXIII a XXVII, t. V, 1866, 153-165.

82. Dante Alligheri [Alighieri], La divina comedia. El purgatorio. Cantos de XXVIII a XXXIII y último, t. V, 1866, 193-208.

83. Dante Alligheri [Alighieri], La divina comedia. El paraíso. Cantos del I a VI, t. V, 1866, 238-252.

84. Dante Alligheri [Alighieri], La divina comedia. El paraíso. Cantos de VII a $X I$, t. V, 1866, 276-288.

85. Dante Alligheri [Alighieri], La divina comedia. El paraíso. Cantos de XII a XVII, t. V, 1866, 317-331.

86. Dante Alligheri [Alighieri], La divina comedia. El paraíso. Cantos de XVIII a XXII, t. V, 1866, 399-411.

87. Dante Alligheri [Alighieri], La Divina Comedia. El Paraíso. Cantos de XXIII a XXVIII, t. VI, 1870, 1-16.

88. Dante Alligheri [Alighieri], La Divina Comedia. El Paraíso. Cantos de XXIX a XXXIII y último, t. VI, 1870, 73-85.

89. Eckerman, J[uan] P[edro] [Eckermann, Johann Peter], "Recuerdos de Goethe”. Memorial, t. II, 1863, 222-224.

90. [ERCILla, Alonso DE], Poetas antiguos españoles. Elección de un caudillo [fragmento de La Araucana], t. II, 1863, 190-192.

91. Fernández Matheu, J[osé], Las baladas de Schiller, t. VI, 1870, 117-121.

92. Feuerbach, Luis [Ludwig], Aforismos, trad. Juan Font y Guitart, t. I, 1862, 159160.

93. Feulllet, Octavio [Octave], Escenas de la vida parisiense. La primera cana, t. VI, 1870, 185-189.

94. FOnT y GUITART, JUAN, Alejandro de Humboldt, t. I, 1862, 122-138.

95. FONT y GUITART, JUAN, Humboldt y el Austria, t. I, 1862, 140-141.

96. Franzen, S. [ADAm Wilhelm (?)], La faz del hombre, t. II, 1863, 231-232.

97. Freiligrath, F[ERnAndo] [Ferdinand], Cabalgata del León, t. I, 1862, 474.

98. García de VillanueVa, M[ANUEL], Historia. El teatro y las fiestas públicas. I, t. VI, 1870, 135-141.

99. García de Villanueva, M[Anuel], Historia. El teatro y las fiestas públicas. II, t. VI, 1870, 153-159.

100. García de Villanueva, M[ANUel], Historia. El teatro y las fiestas públicas. III, t. VI, 1870, 193-199. 
101. García de VillanueVa, M[ANUel], Historia. El teatro y las fiestas públicas. IV, t. VI, 1870, 233-240.

102. García de VillanueVa, M[ANUel], Historia. El teatro y las fiestas públicas. $V$, t. VI, 1870, 273-278.

103. García de VillanueVa, M[ANUel], Historia. El teatro y las fiestas públicas. VI, t. VI, 1870, 315-323.

104. García de VillanUeVA, M[ANUEL], Historia. El teatro y las fiestas públicas. VII, t. VI, 1870, 353-363

105. Gerstemberg [Gerstenberg, Heinrich Wilhelm], Ditirambo, trad. J[osé] Fernández Matheu, t. V, 1866, 359-360.

106. [Gerstenberg, Heinrich Wilhelm], Inmortalidad, trad. J[osé] Fernández Matheu, t. VI, 1870, 232.

107. [GeSSner, SAlomon], Poesías alemanas. Milon, trad. J[osé] Fernández Matheu, t. V, 1866, 471.

108. [GeSSner, SAlOMON], Poesías alemanas. La promesa, trad. J[osé] Fernández Matheu, t. V, 1866, 472.

109. GEÜER, N., El rey del mar, t. II, 1863, 311-312.

110. GoETHE, [JOHANN WolfGANG], Última meditación. Los naturalistas franceses, t. II, $1863,10-13$.

111. Goethe, [Johann WolfGANG], Fausto, t. IV, 1865, 96-99.

112. Goethe, [Johann WolfGANG], Fausto. II, t. IV, 1865, 142-151.

113. Goethe, [Johann WolfGAng], Fausto. III, t. IV, 1865, 182-186.

114. Goethe, [Johann WolfGAng], Fausto. IV, t. IV, 1865, 222-232.

115. Goethe, [Johann WolfGang], Fausto. V, t. IV, 1865, 258-269.

116. GoEThe, [JohANn WolfGAng], Fausto. IV [corresponde al VI]. Segunda parte de la tragedia, t. IV, 1865, 287-302.

117. Goethe, [Johann WolfGang], Fausto. VII, t. IV, 1865, 334-349.

118. Goethe, [Johann Wolfgang], Fausto VIII. Segunda parte de la tragedia, t. IV, $1865,371-382$.

119. Goethe, [Johann Wolfgang], Fausto. IX, t. IV, 1865, 413-421.

120. [GoEThe, JohAnN WolfGAnG], El amante multiforme, trad. T. Llorente, t. IV, $1865,436$.

121. Goethe, [Johann WolfGang], Fausto. $X$, t. IV, 1865, 449-457.

122. GoEThe, [JohANN WolfGANG], Fausto. XI y último. Tercera parte de la tragedia, t. V, 1866, 4-7. 
123. Goethe, [Johann WolfGang], Werther, t. V, 1866, 61-70.

124. Goethe, [JohAnN WolfGang], Werther, t. V, 1866, 104-112.

125. Goethe, [JohANn WolfGang], Werther, t. V, 1866, 185-192.

126. Goethe, [Johann WolfGang], Werther, t. V, 1866, 231-232.

127. Goethe, [JohAnN WolfGang], Werther, t. V, 1866, 270-272.

128. Goethe, [Johann WolfGang], Werther, t. V, 1866, 308-311.

129. Goethe, [JohANn WolfGANG], Werther, t. V, 1866, 348-351.

130. [Goethe, Johann Wolfgang], Poesía alemana. La inconstancia, trad. J[osé] Fernández Matheu, t. V, 1866, 431.

131. [Goethe, Johann Wolfgang], Poesías alemanas. Canción de mayo, trad. J[osé] Fernández Matheu, t. V, 1866, 471-472.

132. [Goethe, Johann Wolfgang], La leyenda del Pavia, trad. J[osé] Fernández Matheu, t. VI, 1870, 151-152.

Goethe: v. también ECKeRManN, J. P.

133. GRIMM, [JAKOB y WiLhELM], El rey barba aguda, trad. José S. Biedma, t. V, $1866,430-431$.

134. Grimm, [JAKoB y Wilhelm], La Paja, el Carbón y el Haba, trad. José S. Viedma [sic], t. V, 1866, 470.

135. [Grimm, JAKOB y Wilhelm], Ruiponche (Cuento de Grim [sic]), trad. José S. Biedma, t. VI, 1870, 71-72.

136. Grimm, [JAKob y Wilhelm], El Doctor Cangrejo, trad. Emilio Mata, t. VI, 1870, 151.

137. Gruneisen, C[arlos] [Grüneisen, Karl Christian Heinrich (?)], El viagero [sic], t. II, 1863, 107-108.

138. Gunther, J[UAN] C[Ristián] [Günther, Johann Christian], Consuelo, t. II, 1863, 185-186.

HALBSUter, JohANNES: v. SUTER, HALB.

139. HaRdenberG, F[EDERICO] DE [HARDENBERG, FriedRICH VON, seud.: Novalis], El trovador, t. II, 1863, 186.

140. Hebel, [Johann Peter], Encuentro inesperado, trad. Antonio Bergnes de las Casas, t. I, 1862, 36.

141. Hebel, [Johann Peter], Kannitverstan, trad. J. S. P., t. I, 1862, 239-240.

142. Hebel, J[UAN] P[EDRO] [JohANN PETER], La tempestad, t. II, 1863, 30. 
143. HeIne, EnRIQUe [HeInRICH], Wenn ich in deine Augen seh'; Du schoenes Fischermädchen; Du bist wie eine Blume; Es liegt der heisse Sommer; Sag wo ist dein schönes Liebchen; Auf Flügeln des Gesanges; Ich weisz nicht was soll es bedeuten / La Lore-Lay, trad. Juan Font y Guitart, t. I, 1862, 39-40.

144. [HeINE, HeInRICH], Poesías de Enrique Heine [incluye: Du schönes Fischermädchen; Du bist wie eine Blume; Lieb Liebchen leg's Händchen auf's Herze mein; Und als ich euch meine Schmerzen geklagt; Verreith mein blasses Angesicht; Wenn ich auf dem Lager liege; Wie der Mond sich leuchtend dränget; Sie liebten sich Beide, doch Keiner; Mir träumte von einem Königskind; Die blauen Veilchen der Aeugelein; Mir träumte: schaurig schaute der Mond; Ueber die Berge steigt schon die Sonne; Sie gaben mir Rath und gute Lehren / Coronación; Meeresstille! ihre Strahlen / Mar Bonanz; Hoffnung und Liebe! alles zertrümmert / Naufragio], trad. Juan Font y Guitart, t. I, 1862, 78-79.

145. Heine, E[nRique] [Heinrich], Don Ramiro, t. II, 1863, 431-432.

146. [Heine, Heinrich], Poesías alemanas. Los besos, trad. J[osé] Fernández Matheu, t. V, $1866,472$.

147. Herder, [Johann GotTfried], Los tres amigos, trad. Antonio Bergnes de las Casas, t. I, 1862, 35.

148. Herder, [Johann GotTtrRIED], El hijo de la Misericordia, trad. J. S. P., t. II, 1862, 240.

149. Herder, [Johann GotTfried], La luz del hogar, trad. J. S. P., t. I, 1862, 240.

150. HeRder, [JohANn GotTFried], La Rosa, t. I, 1862, 474.

151. Hoelty, B. [Hölty, Ludwig Christoph HeinRICH], La vida campestre, t. II, $1863,310-311$.

152. Hoelty [HÖlty, Ludwig Christoph Heinrich], Noche de Mayo, trad. J[osé] Fernández Matheu, t. VI, 1870,32.

153. HoffMANn, [ERnSt TheOdor AmAdeUs], Un aficionado a puntos de vista, t. I, $1862,319-320$.

154. [Humboldt, AleXANDER von], Una carta de Humboldt, trad. Juan Font y Guitart, t. I, 1862, 139-140.

155. IMMERMANN, C[ARLOS] [KARL LEBERECHT], El pescador, t. II, 1863, 109.

156. IRVING, WASHINGTON, El barón Von-Koëldwethout de Tronsberg, t. I, 1862, 396 400.

157. JACOB, P. L. [seud. de LACROIX, PAUL (?)], Estudios morales. Un quebrado. 1777, t. IV 1865, 392-396.

158. JACOB, P. L. [seud. de LACROIX, PAUL (?)], Estudios morales. Un quebrado. II y último, t. IV, 1865, 428-432. 
159. JAL, A[Ugusto] [Auguste], La muerte del marinero. Escenas de la vida marítima, t. II, 1863, 305-309.

160. JANER, F[LORENCIO], La poesía española de la Edad media, y la influencia que en ella ejerció el marqués de Santillana, t. I, 1862, 421-424.

161. Janin, J[Ulio] [JUles GABRIEL], Hoffman y Paganini. (Fantasía), t. III, 1864, 109112.

JEAN PAUl: v. RICHTER, JEAN PAUL FRIEDRICH.

162. KLopSTOCK, [FRIEDRICH GoTTLIEB], La Mesíada. I, t. I, 1862, 269-274.

163. KLOPSTOCK, [Friedrich GotTlieB], La Mesíada. II, t. I, 1862, 312-319.

164. KLOPSTOCK, [FRIEDRICH GotTLIEB], La Mesíada. III, t. I, 1862, 349-354.

165. KLOPSTOCK, [FRIEDRICH GoTTLIEB], La Mesíada. IV, t. I, 1862, 385-394.

166. KLopstock, [Friedrich GotTlieB], La Mesíada. V, t. I, 1862, 462-467.

167. Klopstock, [Friedrich GotTlieB], La Mesíada. VI, t. II, 1863, 17-25.

168. Klopstock, [Friedrich GotTlieB], La Mesíada. VII, t. II, 1863, 46-54.

169. KLOPSTOCK, [FRIEDRICH GotTlieB], La Mesíada. VIII, t. II, 1863, 95-100.

170. KLOPSTOCK, [Friedrich GotTlieB], La Mesíada. IX, t. II, 1863, 142-147.

171. KLOPSTOCK, [Friedrich GotTlieB], La Mesíada. X, t. II, 1863, 177-183.

172. KlOPSTOCK, F[EDERICO] [FrIEDRICH GoTTlieB], Hermann y Thusnelda, t. II, 1863, 231.

173. KLOPSTOCK, [Friedrich GotTlieB], La Mesíada. XI, t. II, 1863, 260-271.

174. Klopstock, [Friedrich GotTlieB], La Mesíada. XII, t. II, 1863, 296-303.

175. Klopstock, [Friedrich GotTlieb], La Mesíada. XIII, t. II, 1863, 337-344.

176. Klopstock, [Friedrich GotTlieB], La Mesíada. XIV, t. II, 1863, 378-388.

177. Klopstock, [Friedrich GotTlieB], La Mesíada. XV, t. II, 1863, 454-467.

178. Klopstock, [Friedrich GotTlieB], La Mesíada. XVI, t. III, 1864, 18-25.

179. KLOPSTOCK, [Friedrich GotTLIEB], La Mesíada. XVII, t. III, 1864, 62-68.

180. KLOPSTOCK, [FRIEDRICH GotTLIEB], La Mesíada. XVIII, t. III, 1864, 98-104.

181. KLOPSTOCK, [FRIEDRICH GotTlieB], La Mesíada. XIX, t. III, 1864, 176-183.

182. Klopstock, [Friedrich GotTlieb], La Mesíada. XX y último, t. III, 1864, 250 260.

183. [Klopstock, Friedrich GotTlieb], Poesías alemanas. No me olvides, trad. J[osé] Fernández Matheu, t. V, 1866, 471. 
184. KOERnER, J. [Christian GotTFried (?)], El abeto y la vid, t. III, 1864, 112.

185. KRUMmaCher, [Friedrich Adolf (?)], Los dos ángeles, trad. Antonio Bergnes de las Casas, t. I, 1862, 35.

186. KRUMMACHER, [FRIEDRICH AdOLF (?)], La lucha de fieras, t. I, 1862, 360.

187. KRUMmacher, [Friedrich Adolf (?)], Adán y el Ángel, trad. E[milio] Mata, t. V, $1866,471$.

LACRoIX, PAUl: v. JACOB, P. L. (seud.)

188. Lalande, J., La promesa del Diablo (Cuento fantástico), t. I, 1862, 274-276.

189. LALANDE, J., El poeta, t. II, 1863, 151-152.

190. LARra, Mariano José De, Poesía. Elegía, t. IV, 1865, 275-276.

191. LESSING, G[OTTHOLD] E[PHRAIM], La estatua de bronce, t. III, 1864, 152.

192. LONG FELLON [LONGFELlOW, HENRY WADSWORTH], La copa del rey Witlaff, t. VI, 1870, 190.

193. LONGFELlow, [HENRY WADSWORTH], Los Cantores, t. VI, 1870, 272.

194. MACÍAS, F[ELIPE] A., Ensayo sobre las condiciones literarias y morales del refrán español, traído a mas regulares formas. I, t. V, 1866, 341-342.

195. MACÍAS, F[ELIPE] A., Ensayo sobre las condiciones literarias y morales del refrán español traído a más regulares formas, t. V, 1866, 388-392.

196. MACÍAS, Felipe A., Filología. Examen crítico de los sistemas de escritura, t. VI, 1870, 62-64.

197. MACÍAS, FELIPE A., Literatura. El ritmo de la frase, t. VI, 1870, 104-107.

198. MAC-PHERSON [MACPHERSON, JAMES], Estudios crítico-literarios. Sobre los poemas de Osian [sic]. I, t. II, 1863, 293-296.

199. MAC-Pherson [MACPHerson, JAMES], Estudios crítico-literarios. Sobre los poemas de Osian [sic]. II. t. II, 1863, 334-336.

200. MAC-Pherson [MAcPHerson, JAmes], Estudios crítico-literarios. Sobre los poemas de Osian [sic]. III, t. II, 1863, 376-377.

201. MAC-PHERSON [MACPHERSON, JAMES], Estudios crítico-literarios. Sobre los poemas de Osian [sic]. IV, t. II, 1863, 426-430.

202. MAC-PHERSON [MACPHERSON, JAMES], Estudios crítico-literarios. Sobre los poemas de Osian [sic]. V, t. III, 1864, 68-71.

203. MAC-Pherson [MACPHERSON, JAMES], Estudios crítico-literarios. Sobre los poemas de Osian[sic]. VI y último, t. III, 1864, 218-222.

MACPHERSON, JAMES: v. también OSSIAN (seud.) 
Mendoza de Vives, María: v. Vives, María Mendoza de.

204. MenZel, WolfGang, La estación de las rosas, t. III, 1864, 152.

205. Metastasio, [Pietro], El amor prisionero, trad. de R. F. B., t. VI, 1870, 313-314.

206. [Milton, John], Cántico de Milton. La fiesta de Navidad, trad. J[osé] Fernández Matheu, t. V, 1866, 360.

207. MiR-HASSAN-Alí, Vida oriental, t. I, 1862, 262-269.

208. Montesquieu, [CHARles], Cefisa y amor, trad. A[urelio] Queralt [sic], t. V, $1866,473$. 12.

209. Muller, [CARlos] [Müller, KARl (?)], Juan Cristiano Oersted, t. I, 1862, 11 -

210. Muller, Carlos [Müller, Karl (?)], El sastre chino, trad. Antonio Bergnes de las Casas, t. I, 1862, 97-98.

NOVAlis (seud.): v. HARDENBERG, FRIEDRICH VON.

211. Nuszlein, F[RANCISCO] A[NTONIO] [NÜSSLEIn, Franz ANTON], Filosofía ideal. Estética. I, t. III, 1864, 1-5.

212. Nuszlein, F[rancisco] A[NTONiO] [NÜsslein, Franz Anton], Filosofía ideal. Estética. II, t. III, 1864, 33-37.

213. Nuszlein, F[rancisco] A[ntonio] [NÜsslein, Franz Anton], Filosofía ideal. Estética. III, t. III, 1864, 73-79.

214. Nuszlein, F[RANCISCO] A[NTONio] [NÜSSlein, FrAnz Anton], Filosofía ideal. Estética. IV, t. III, 1864, 113-118.

215. Nuszlein, F[rancisco] A[ntonio] [Nüsslein, Franz Anton], Filosofía ideal. Estética. V, t. III, 1864, 153-163.

216. Nuszlein, F[RAnCISCO] A[NTONio] [NÜSSlein, Franz Anton], Filosofía ideal. Estética. VI, t. III, 1864, 193- 197.

217. Nuszlein, F[RANCISCO] A[NTONiO] [NÜSSLEIn, Franz Anton], Filosofía ideal. Estética. VII, t. III, 1864, 233-236.

218. Nuszlein, F[RAnCisco] A[NTONio] [NüSslein, Franz Anton], Filosofía ideal. Estética. VIII, t. III, 273-279.

219. Nuszlein, F[rancisco] A[ntonio] [Nüsslein, Franz Anton], Filosofía ideal. Estética. IX, t. III, 1864, 313-319.

220. Nuszlein, F[RAnCisco] A[NTOnio] [NÜSSlein, Franz Anton], Filosofía ideal. Estética. $X$, t. III, 1864, 353-362.

221. Nuszlein, F[rancisco] A[NTOnio] [NüSslein, Franz Anton], Filosofía ideal. Estética. XI, t. III, 1864, 393-398. 
222. Nuszlein, F[RAnCisco] A[NTOnio] [NÜsslein, Franz Anton], Filosofía ideal. Estética. XII, t. III, 1864, 433-439.

223. Nuszlein, F[RAncisco] A[ntonio] [NÜsslein, Franz Anton], Filosofía ideal. Estética. XIII, t. IV, 1865, 5-11.

224. Nuszlein, F[RANCISCO] A[NTONiO] [NÜSSlein, FRAnZ ANTON], Filosofía ideal. Estética. XIV, t. IV, 1865, 77-81.

225. Nuszlein, F[RANCISCO] A[NTONiO] [NÜSSlein, Franz ANTON], Filosofía ideal. Estética. XV y último, t. IV, 1865, 117-120.

226. Osian [Ossian, seud. de Macpherson, James], Témora. Canto I, t. III, 1864, 262-267.

227. Osian [Ossian, seud. de Macpherson, James], Témora. Canto II, t, III, 1864, 296-300.

228. OSian [OSSIAn, seud. de MACPherson, James], Témora. Canto III, t. III, 1864, 344-348.

229. Osian [OSSIAN, seud. de MaCPherson, James], Témora. Canto IV, t. III, 1864, 371-375.

230. Osian [OsSian, seud. de MacPherson, JAMES], Témora. Canto V, t. III, 1864, 410-413.

231. Osian [Ossian, seud. de Macpherson, James], Témora. Canto VI, t. IV, 1865, 69-73.

232. Osian [Ossian, seud. de Macpherson, James], Témora. Canto VII, t. IV, 1865, 270-273.

233. Osian [OSSIAN, seud. de MACPHERSon, JAmes], Témora. Canto VIII y último, t. IV, 1865, 352-356.

234. Osian [OSSIAN, seud. de MACPHERSon, JAmes], La muerte de Cutulino. Canto único, t. IV, 1865, 383-386.

OSSIAN (seud.): v. también MACPHERSON, JAMES.

235. Pfeffel, [GotTlieb KonRAD], La oveja perdida, t. I, 1862, 359-360.

236. PfeFfel, A[MADEO] C[ONRADo] [GotTlieb KonRAD], La dama blanca, t. II, 1863, 469-471.

237. Platón, El Phedón, o la inmortalidad del alma. I, t. II, 1863, 273-276.

238. Platón, El Phedón, o de la inmortalidad del alma. II y último, t. II, 1863, $320-$ 324.

239. REINICK, J. [REINICK, ROBERT (?)], Alda, t. II, 1863, 232. 
240. Richter, Juan Pablo federico [Jean Paul Friedrich, seud.: Jean Paul], Sueño del entierro. El doble juramento de enmienda, trad. Antonio Bergnes de las Casas, t. I, 1862, 37-39.

241. Richter, Juan Pablo F[EDerico] [JeAn Paul Friedrich, seud.: Jean PaUl], El sueño de una loca, trad. Juan Font y Guitart, t. I, 1862, 190-192.

242. Richter, Juan Pablo Federico [Jean Paul Friedrich, seud.: JeAn PAUl], Recuerdos de las horas más bellas para las postreras de la vida, trad. Antonio Bergnes de las Casas, t. I, 1862, 193-198.

243. Richter, Juan Pablo Federico [Jean Paul Friedrich, seud.: Jean PaUl], Extractos [incluye: Aniquilación; El campo de batalla, - melancolía, - recuerdos], trad. Antonio Bergnes de las Casas, t. I, 1862, 232-239.

244. Richter, [JEAN PAUl Friedrich, seud.: JEAn PAUl], Noche de año nuevo de un desgraciado, t. I, 1862, 438-439.

245. [RICHTER, JEAN PAUl FRIEDRICH, seud.: JEAN PAUL], Un desenlace inesperado, t. I, 1862, 439-440.

246. Richter, J[UAN] P[ABLo] F[EDerico] [JEAn PAUl Friedrich, seud.: JeAn PAUl], La azucena del valle, t. II, 1863, 150.

247. RICHTER, J[UAN] P[ABLO] F[EDERICO] [JEAN PAUl FRIEDRICH, seud.: JEAN PAUL], El habla de amor, t. II, 1863, 231.

248. Richter, F[ederico] [Jean Paul Friedrich, seud.: JeAn PaUl], Visión, trad. J[osé] F[ernández] M[atheu], t. V, 1866, 473-474.

249. Royer, A[LFOnso] [Alphonse], El clavo de Zahed. Oriental, t. III, 1864, 385392.

250. RUCKERT, F[EDERICO] [RÜCKERT, FRIEDRICH], Federico Barbarroja, t. II, 1863, 150.

251. Saintine, J[AVIER] B[Onifacio] [Boniface Saintine, XAVIER], El hijo del hechicero, t. II, 1863, 225-228.

252. SAINTINE, J[AVIER] B[ONIFACIO] [BONIFACE SAINTINE, XAVIER], La isla del cocotero, t. III, 1864, 25-28.

253. SAInT-ReAl, [CÉSAR Vichard, ABbÉe DE SAINT-RÉAL], Discurso sobre la belleza de las mugeres. I, t. V, 1866, 465-470.

254. SAINT-REAL, [CÉSAR VICHARD, ABbÉE DE SAINT-RéAL], Discurso sobre la belleza de las mugeres. II, t.VI, 1870, 30-32.

255. SAInT-ReAl, [CÉSAR Vichard, ABbée DE SAINT-RÉAL], Discurso sobre la belleza de las mugeres. III y último, t. VI, 1870, 52-56.

256. SAURA, S[ANTIAGO] Á[NGEL], El carnaval en la antigüedad y en la edad media. I. Tiempos antiguos, t. II, 1863, 42-45. 
257. SAURA, S[ANTIAGO] Á[NGEL], El carnaval en la antigüedad y en la edad media. II y último. Edad media, t. II, 1863, 82-85. 72.

258. SaURa, S[ANTIAGO] Á[NGEL], Fantasía religiosa. Luz y tinieblas, t. III, 1864, 71-

259. SCHILlER, [FRIEDRICH], El criminal por la honra perdida, trad. Antonio Bergnes de las Casas, t. I, 1862, 28-35.

260. SCHILLER, [FRIEDRICH], El teatro considerado como una institución moral, trad. Juan Font y Guitart, t. I, 1862, 117-120.

261. SCHILler, Fed[ERICO] [FRIEDRICH], Al placer. Canto único, t. II, 1863, 30-31.

262. SCHILLER, FED[ERICO] [FRIEDRICH], La campana, t. II, 1863, 148-150.

263. SCHILLER, F[EDERICO] [FRIEDRICH], Las tres palabras de la Fe, t. II, 1863, 467.

264. [SChiller, Friedrich], Poeśía. Éxtasis, trad. Teodoro Llorente, t. IV, 1865, 435.

265. SCHILLER, [FRIEDRICH], María Estuardo, t. V, 1866, 19-27.

266. SCHILLER, [FRIEDRICH], María Estuardo. Acto segundo, t. V, 1866, 49-56. 72.

267. [SCHILleR, FRIEDRICH], El anillo de Polícrates, trad. Teodoro Llorente, t. V, 1866,

268. SCHILLER, [FRIEDRICH], María Estuardo. Acto tercero, t. V, 1866, 89-94.

269. SCHILlER, [FRIEDRICH], Las palabras de la ilusión, trad. Carlos Medina, t. V, $1866,112$.

270. SChiller, [Friedrich], María Estuardo. Acto cuarto, t. V, 1866, 130-136. 152.

271. [SChILleR, Friedrich], Poesía. El guante, trad. Teodoro Llorente, t. V, 1866,

272. SCHILleR, [FrIEDRICH], María Estuardo. Acto quinto, t. V, 1866, 165-171.

273. Schiller, [Friedrich], Canto de Victoria, trad. José Fernández Matheu, t. V, 1866, 297-298.

274 SCHILler, [FRIEDRICH], El cazador de los Alpes, trad. José Fernández Matheu, t. $\mathrm{V}, 1866,312$.

275. [SChiller, Friedrich], Poesía alemana [incluye: Canción de las montañas, La fortuna y la prudencia, Sentencia de Confucio], trad. J[osé] Fernández Matheu, t. V, 1866, 431.

276. [SCHILlER, FRIEDRICH], El homenaje a las artes. Composición dramáticà de F. Schiller, trad. Emilio Mata, t. V, 1866, 458-460.

277. [SCHILleR, FriedRICH], Poesías alemanas. La esperanza, trad. J[osé] Fernández Matheu, t. V, 1866, 472. 
278. [SChiller, Friedrich], Poesías alemanas. La luz y el calor, trad. J[osé] Fernández Matheu, t. V, 1866, 472.

279. [SCHILLER, FRIEDRICH], El conde Eberhard de Wurtemberg; El caballero Toggenbürg, trad. J[osé] Fernández Matheu, t. VI, 1870, 121.

280. SCHILLER, [FRIEDRICH], A Laura, t. VI, 1870, 270-271.

281. SCHILlER, [FRIEDRICH], A Laura. Reminiscencias, t. VI, 1870, 271-272.

282. [SChleiermacher, Friedrich], El Mundo. De los Monólogos de Schleiermacher, trad. Antonio Bergnes de las Casas, t. I, 1862, 186-189.

283. Schubart, C[RISTIÁn] F[EDERICo] [Christian Friedrich], El panteón de los príncipes, t. II, 1863, 29-30.

284. Scott, Walter, Amor y gloria. Poema, t. IV, 1865, 459-469.

285. SEIDL, C., La campanilla de la felicidad, t. II, 1863, 108-109.

286. Simonet, F[RANCISCO] J[AVIER], El judio Samuel Ebn'Adia, t. I, 1862, 475-479.

287. Stolberg, F[ederico] L[Eopoldo] De [Friedrich Leopold, GraF ZU], El torrente del monte, t. III, 1863, 150-151.

288. SuART, M., Biografía. Torcuato Tasso, t. III, 1863, 25-28.

289. Sue, E[UGENIO] [EugÈnE], El capitán Wolf. I, t. IV, 1865, 73-76.

290. Sue, E[UGENIO] [EugÈnE], El capitán Wolf. II y último, t. IV, 1865, 189-192.

291. Suter, Halb [HAlbSUTER, JohANNES], La batalla de Sempach, t. II, 1863, 28-29.

292. [SYV, PEDER P.], Langben el gigante y Vidrik hijo de Verland [trad. del danés al alemán por W. Grimm en sus Daenische Heldenlieder], t. II, 1863, 473-474.

293. TIECK, L[UIS] [LUDWIG], La primavera, t. II, 1863, 72.

294. [Uhland, Ludwig], Poesías de Luis Uhland [incluye: La ermita; Despedida; Sones de muerte], trad. Juan Font y Guitart, t. I, 1862, 80.

295. [Uhland, Ludwig], Poesías alemanas. Las palabras del anciano, trad. J[osé] Fernández Matheu, t. V, 1866, 472.

296. [Valmiki], El Ramayana. Poema sánscrito. Traducido y reducido de las versiones hechas por algunos orientalistas modernos. I, trad. D. P. Puigbó, t. V, 1866, 362-366.

297. VAlmiki, El Ramayana. Poema sánscrito. II, t. V, 1866, 440-445.

298. Valmiki, El Ramayana. Poema sánscrito. III, t. VI, 1870, 46-50.

299. VAlmiki, El Ramayana. Poema sánscrito. IV t. VI, 1870, 124-128.

300. VAlmiki, El Ramayana. Poema sánscrito. V, t. VI, 1870, 165-169.

301. VAlmiki, El Ramayana. Poema sánscrito. VI, t. VI, 1870, 204-208. 
302. VAlmiki, El Ramayana. Poema sánscrito. VII, t. VI, 1870, 242-246.

303. VAlmiki, El Ramayana. Poema sánscrito. VIII, t. VI, 1870, 281-285.

304. VAlmiki, El Ramayana. Poema sánscrito. IX, t. VI, 1870, 332-337.

305. VALMIKI, El Ramayana. Poema sánscrito.VII, t. VI, 1870, 372-376.

306. Vives, María Mendoza de [Mendoza de Vives, María], El amor de los amores. Leyenda sacada de una balada alemana, t. I, 1862, 198-200.

307. WERNER, Z[ACARÍAS] [ZACHARIAS], La dicha, t. II, 1863, 432.

308. Wessenberg, J. H. DE [IgnaZ HeinRICH Von], El juicio final. Dies irae, t. II, $1863,474$.

\section{2. 2. Índice de traductores.}

a. BERGNES DE LAS CASAS, ANTONIO: números 44, 49, 62, 140, 147, 185, 210, 240 , 242, 243, 259, 282.

b. BIEDMA, JosÉ S.: números 133, 134, 135.

c. Fernández MATHeU, JosÉ: números 66, 105, 106, 107, 108, 130, 131, 132, 146, $152,183,206,248,273,274,275,277,278,279,295$

d. FONT Y GUITART, JUAN: números 92, 143, 144, 154, 241, 260, 294.

e. J. S. P.: números 141, 148, 149.

f. Llorente, TeOdoRo: números 120, 264, 267, 271.

g. MATA, EMilio: números 137, 187, 276.

MAtheU, J. F.: v. Fernández MatheU, José.

h. MAUPOEY, ENRIQUE: número 60.

i. Medina, CARlos: número 269.

j. PuigBó, D. P.: números 296-305.

QUERAL, AURELIO: v. QUEROL, AURELIO.

Queralt, A.: v. QUeRol, AURElio.

k. Querol, Aurelio: números 48, 68, 208.

1. R. F. B.: número 205.

VIEDMA, José S.: v. BIEDMA, JosÉ S. 
